\title{
The metric of visual space
}

\author{
MARK WAGNER \\ Franklin and Marshall College, Lancaster, Pennsylvania
}

\begin{abstract}
The geometric properties of visual space in the horizontal plane were measured by four different procedures. Five observers were asked to judge distances, angles, and areas defined by pairs and triplets of stakes, using magnitude estimation, category estimation, mapping, and perceptual matching. All judgments took place outdoors in a broad, open field under full-cue conditions. Stimuli oriented in depth were judged to be half as large as the same stimuli oriented in the frontal plane. Angles facing either directly toward or directly away from the observer were seen as approximately twice as large as those seen on their sides. Four mathematical models for visual space are examined. Both the hyperbolic model of Luneburg (1947) and the spherical model of Reid (1764/1813) fail, each for different reasons. Two other models, however, produce a reasonably complete description of visual space. In the first model, visual space is an affine-transformed version of a Euclidean physical space. In the second model, distances are viewed as vectors that can be broken down into in-depth and frontal components relative to the observer. The in-depth component of this vector is contracted by a constant amount in visual space.
\end{abstract}

The geometric structure of visual space has long been an object of controversy in perceptual psychology. Many synthetic accounts have been proposed for visual space: Gibson $(1950,1959)$ states that visual space is Euclidean; Angell (1974), Daniels (1974), and Reid (1764/1813) suggest it is spherical; Blank $(1953,1959,1978)$, Indow (1967, 1974, 1979), and Luneburg $(1947,1950)$ propose it is hyperbolic; Hoffman $(1966,1980)$ believes it reflects a Lie algebra group.

This paper presents a systematic investigation of judgments of distances, angles, and areas-the metric properties of a space. The examination of several metric properties simultaneously is interesting in itself, since it has seldom been attempted in the past. More important, however, since the metric properties of the synthetic geometries may be derived, this analytic approach can provide a direct test for the previous, synthetic work, as Luneburg (1948) himself suggested. In fact, by knowing the metric properties and their interrelations, we actually define the geometry of visual space analytically without recourse to the general and largely untestable assumptions that characterize a synthetic geometry.

The empirical basis of this analysis is visual psychophysics. Most psychophysical studies rely upon a single method (Baird, 1970). When only a single method is applied, it is impossible to know whether the resulting psychophysical function is due to perception itself or to response processes inherent in the method. The present work applies several psychophysical techniquesmagnitude estimation, category estimation, perceptual

This article is based upon a doctoral dissertation completed at Dartmouth College. I would like to thank John C. Baird and Hiroshi Ono for their helpful comments on this paper. Requests for reprints should be sent to Mark Wagner. Department of Psychology. Franklin and Marshall College, P.O. Box 3003, Lancaster, PA 17604-3003. matching, and mapping. Commonalities resulting from these converging measures should reflect fundamental aspects of visual space that are independent of the methods employed.

\section{METHOD}

\section{Observers}

Five observers participated in each of the four experimental phases. Three of the observers were naive regarding psychophysical scaling techniques; 2 were familiar with their use and interpretation ( 1 observer was the experimenter).

\section{Apparatus and Layout}

The primary apparatus consisted of 13 stakes. A whitewashed board (25.5 $\mathrm{cm}$ high, $18 \mathrm{~cm}$ wide, and $100 \mathrm{~cm}$ above the ground) was attached to the top of each. Each stake was uniquely identified by a black letter (approximately $23 \mathrm{~cm}$ high and $14 \mathrm{~cm}$ wide) affixed to the center of the board.

The experiment took place outdoors in a large, flat open field. The grass was uncut but relatively short, providing abundant textural cues to depth without obscuring the stakes set in the field. Observers, sitting on stools, were allowed to move their heads freely during the process of judgment. Although they were allowed to move within $1 \mathrm{~m}$ of their viewing position, none of the observers took advantage of this opportunity.

Ten stakes were placed randomly in the field, using a uniform distribution subject to three constraints: (1) All stakes were confined to a region in front of the observer $40 \mathrm{~m}$ deep and $40 \mathrm{~m}$ wide; (2) from an origin. $(0.0)$, placed at the middle of the near edge of the stake field, no stakes could deviate by more than $45^{\circ}$ either to the left or the right of straight ahead (Figure 1 displays the region within which all stakes were placed); and (3) no two stakes could be closer than $5 \mathrm{~m}$ from each other. A different stake randomization was employed on each day of the experiment.

Judgments were made from three different origins on the field: The first was coincident with the origin used to define stake locations; the second was $20 \mathrm{~m}$ farther from the stakes; and the third was $20 \mathrm{~m}$ farther from the stakes than the second (see Figure 1).

Three additional stakes served as a reference standard and were placed $10 \mathrm{~m}$ to the observer's right. The base and height of a triangle determined by these three stakes were each $5 \mathrm{~m}$. 


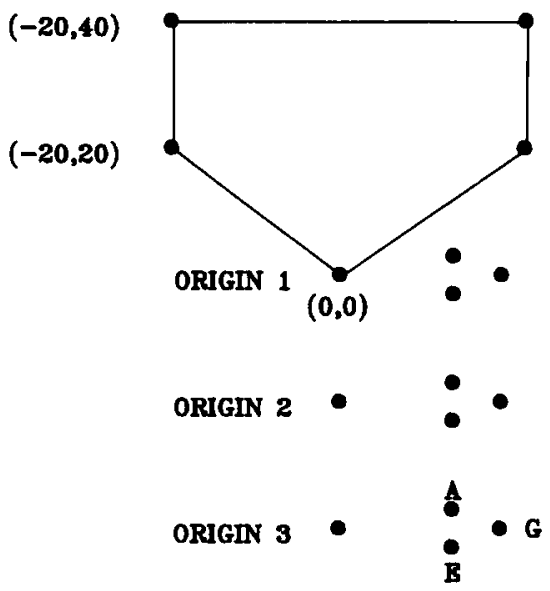

$(20,40)$

Figure 1. Diagram displaying the pentagonal region in which stakes were randomly placed and the three origins from which judgments were made. The three points lying next to each origin represent the standard for that origin. Numbers in parentheses are the Cartesian coordinates of various points in meters.

\section{Procedure}

The experiment took place on 16 consecutive weekdays and was divided into four distinct phases involving the four different judgment techniques in the following order: magnitude estimation, category estimation, mapping, and perceptual matching. The subjects were asked to judge objective as opposed to projective sizes (see Baird, 1970, Carlson, 1977, and Leibowitz \& Harvey, 1969, for discussion on the effects of instructions).

During the magnitude estimation phase, judgments were made for three different metric properties of visual space-distance, angle, and area. In the distance estimation phase, the observers were told to assume that the distance between stakes $\mathrm{A}$ and $\mathrm{E}$ (see Figure 1) was 100 arbitrary units-physically $5 \mathrm{~m}$ long. Numerical estimates of distances between a variety of other stake pairs were made with respect to this reference standard. For example, if the distance between stakes $\mathrm{C}$ and $\mathrm{F}$ appeared to be twice as great as that of the standard, the observer would say " 200 ," if it was half as great, the observer would say " 50 ," and so forth. Any number (including fractions) was legitimate. Similar instructions were given for angle estimation, in which the reference standard of 100 units was assigned to the angle AGE, where $G$ was the vertex-physically $53^{\circ}$-and area estimation, in which the reference standard of 100 units was assigned to the triangle with the stakes $A, G$, and $E$ as its vertices-physically $25 \mathrm{~m}^{2}$. A total of 90 distance (all possible pairs repeated twice), 120 angle (all possible angles), and 60 area judgments were made by each observer from each origin. The stimuli ranged between 7 and $72 \mathrm{~m}$ for distance estimation, $1^{\circ}$ and $179^{\circ}$ for area estimation, and 1 and $365 \mathrm{~m}^{2}$ for area estimation.

During the category estimation phase, the observers judged the same three metric properties of visual space as in magnitude estimation. In the distance estimation phase, the observers were asked to imagine the range from the smallest distance encountered in the magnitude estimation phase to the largest distance and to divide this range into seven equal parts, each of which was to be assigned an integer between 1 and 7 inclusive. Similar instructions were given for angle and area estimation. The number of judgments collected for each was the same as in magnitude estimation.

During the mapping phase, each observer was given a sheet of paper and a set of pins. On each pin, a letter was attached corresponding to one of the stakes in the field. The observers were asked to stick the pins in the paper in order to create a map that corresponded to their perception of the stake layout. The observers could orient the paper any way they wished and could adjust the pins until satisifed that the map represented the stake layout. The metric properties subsequently could be read off the maps.

During the perceptual matching phase, the observers were given a set of 19 cards. Angles between $0^{\circ}$ and $180^{\circ}$ in $10^{\circ}$ increments were drawn on each. The observer's task was to match each angle formed by the stakes in the field with the angle on the card that most nearly equaled it. For practical reasons, perceptual matching could not be applied to either distance or area estimation. Each observer made 45 judgments at each origin.

\section{RESULTS}

The definition of a metric on a space (such as distance) involves determining how the metric varies as a function of the location of points in a coordinate space. Many sorts of "coordinatizations" are possible outside of the common Cartesian and polar coordinate systems. The present data are discussed in terms of a slightly different coordinate system for which the coordinate dimensions are easy to interpret and are defined relative to the observer. For distance estimation, the location of a point is described by four coordinates: the distance from the observer to the nearest point $(R)$, the polar angle or the direction the nearest point lay relative to an arbitrarily defined axis to the observer's right $(\theta)$, the orientation of the points with respect to the observer's frontal plane $(\phi)$, and the Euclidean distance between the two points (D) (see Figure 2). For angle estimation, there are six coordinates that describe an angle: the distance from the observer to the vertex of the angle (R), the polar angle between the vertex and an arbitarily defined axis $(\theta)$, the orientation of the angle relative to the observer's frontal plane $(\phi)$, where orientation is defined by the vector that bisects the angle, the length of the two legs of the angle (D1 and D2), and the size of the angle itself (A) (see Figure 3). The coordinate system for area estimation is similar.

S. S. Stevens (1975) demonstrated that judgments of unidimensional stimuli almost universally fit a power function. For distance estimation, Baird (1970) showed that the power function describes estimates of stimuli in both the frontal plane and in depth. Thus, if we examine judged distance as a function of actual distance for points in a given

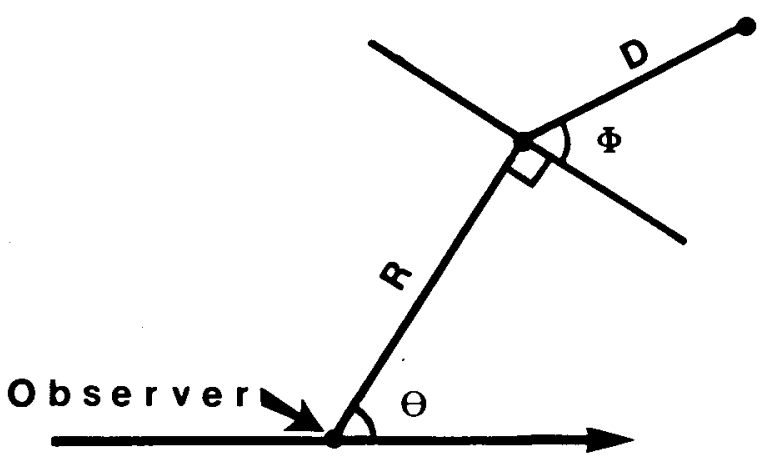

Figure 2. Coordinate system used to specify stake locations for distance estimation. The coordinate dimensions displayed are the radius $(R)$, the polar angle $(\theta)$, the orientation angle $(\phi)$, and the physical interstake distance (D). 


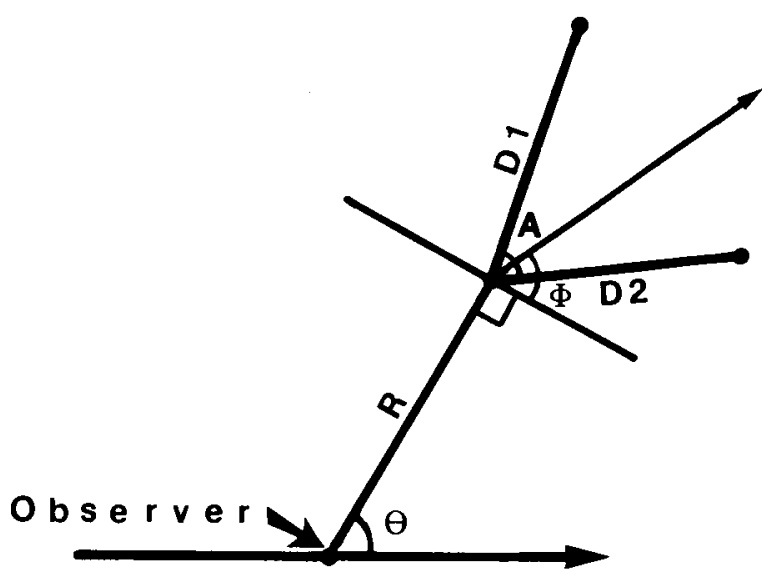

Figure 3. Coordinate system used to specify stake locations for angle estimation. The coordinate dimensions displayed are the radius $(\mathbf{R})$, the polar angle $(\theta)$, the orientation angle $(\phi)$, the lengths of the two legs of the angle (D1 and D2), and the physical size of the angle (A).

location and orientation in space, we would expect to obtain a power function of the form

$$
\mathrm{J}=\mathrm{kD}^{\mathrm{n}} \text {, }
$$

where $\mathrm{k}$ and $\mathrm{n}$ are constants for that particular location and orientation and $\mathrm{J}$ and $\mathrm{D}$ are judged and actual distances, respectively. S. S. Stevens (1975) argues that all unidimensional estimates of the magnitude of stimuli are best fit by a power function irrespective of the judgment method employed (whether magnitude estimation or category estimation, etc.). Different methods, however, will give rise to different power function exponents. Following S. S. Stevens's example, I will use the power function to describe the results from all judgment methods. In this way, cross-method consistency is gained and distortions in judgments due to the response method itself can be accounted for by alterations in the exponent.

The parameters $\mathrm{k}$ and $\mathrm{n}$ need not be the same for all locations and orientations. More specifically, the values of these parameters may vary as a function of spatial location such that

$$
\mathrm{J}=\mathrm{k}(\mathrm{R}, \theta, \phi) \mathrm{D}^{\mathrm{n}(\mathrm{R}, \boldsymbol{\theta}, \boldsymbol{\phi})}
$$

where $\mathrm{R}, \theta$, and $\phi$ are the coordinates mentioned above. A similar function may be determined for angle and area estimation.

Although the idea that $\mathrm{k}$ and $\mathrm{n}$ might be functions of stimulus conditions is not new (J. C. Stevens \& Hall, 1966; J. C. Stevens \& Rubin, 1970; R. Teghtsoonian \& M. Teghtsoonian, 1978), unique features of the current experimental conditions demand the introduction of several new analysis techniques. Unlike most psychophysical studies, the present study used values for the independent variables that were determined by the random selection of the stake locations rather than being ex- perimentally fixed factors. The primary advantage of randomly selecting independent variables (Brunswik, 1944) is that it avoids the arbitrary selection of special stimulus conditions in much the same way that the random selection of subjects controls for special subject characteristics. Three new analyses are applied to this type of data in order to determine the values of $\mathrm{k}$ and $\mathrm{n}$. The first technique (the global analysis) provides overall estimates of the parameters for individuals or for all observers combined. The other two (the binned regression and the modulus analysis) provide estimates of $\mathrm{k}$ or $\mathbf{n}$ as a function of a coordinate variable. A more detailed description of the latter two techniques is given in Appendix A.

Note that the term "modulus" is used to describe k because the most commonly used terms "scaling factor" and "measure constant" are clearly inappropriate to describe a potentially nonconstant function. Since k may be thought of as the number assigned to a "standard" stimulus of 1-unit magnitude, $k$ is thus a modulus for each viewing condition. For this reason, I will use the term "modulus" to describe $\mathrm{k}$ in this paper.

\section{Global Analyses}

Table 1 presents the overall analyses for each method and each metric property (distance, angle, and area). To fit the data to a power function, all log stimulus-log judgment pairs for each observer with all origins combined were regressed. In addition, a composite analysis was performed in which all log stimulus-log judgment pairs with all observers and origins combined were regressed. The slope of this regression provides an estimate for the overall value of the exponent. By itself, the overall value of the modulus is not meaningful. It is not reported in Table 1 .

Several features of the results should be noted. First, the correlation for the regression varies between .65 and .89. These values, though highly significant when compared with chance, are low when compared with those commonly found in unidimensional distance estimation (M. Teghtsoonian \& R. Teghtsoonian, 1969; R. Teghtsoonian \& M. Teghtsoonian, 1970, 1978), where correlations are commonly close to .98 . Why are the correlations so low here? If either the modulus or exponent are functions of coordinate dimensions, then the global analysis attempts to fit a single power function to data better described by a family of power functions. A single power function will not account for the data generated under all conditions, leading to a low correlation.

The exponents obtained are consistent with previous results. The exponent for distance estimation in magnitude estimation is typically close to 1.0 (Baird, 1970). The exponents for area judgments are consistently less than those produced by distance estimation (Baird \& Noma, 1978). Category estimation exponents tend to be less than magnitude estimation exponents (S. S. Stevens, 1975). Due to the constraints involved in producing a map (Baird, Wagner, \& Noma, 1982), exponents for distance and angle estimation in mapping must be close to 1.0 (Baird, 
Table 1

Overall Exponents (Correlations in Parentheses) for Individual Observers and Composite for Each Metric Property Judged and Each Judgment Method

\begin{tabular}{|c|c|c|c|c|c|c|}
\hline \multirow[b]{2}{*}{ Condition } & \multicolumn{5}{|c|}{ Observer } & \multirow[b]{2}{*}{ Composite } \\
\hline & J.B. & L.C. & N.R. & J.C. & M.W. & \\
\hline \multicolumn{7}{|c|}{ Distances } \\
\hline ME & $.98(.86)$ & $.96(.83)$ & $1.05(.79)$ & $.94(.82)$ & $1.00(.78)$ & $.99(.77)$ \\
\hline $\mathrm{CE}$ & $.99(.89)$ & $.72(.80)$ & $1.00(.83)$ & $.89(.84)$ & $.90(.82)$ & $.90(.83)$ \\
\hline MA & $.95(.82)$ & $.99(.82)$ & $.97(.80)$ & $.95(.82)$ & $.93(.73)$ & $.96(.76)$ \\
\hline \multicolumn{7}{|c|}{ Angles } \\
\hline ME & $.74(.83)$ & $.81(.79)$ & $.85(.82)$ & $.79(.86)$ & $.82(.82)$ & $.81(.82)$ \\
\hline CE & $.48(.83)$ & $.50(.76)$ & $.50(.79)$ & $.45(.81)$ & $.44(.78)$ & $.47(.78)$ \\
\hline MA & $.95(.81)$ & $.85(.79)$ & $1.07(.77)$ & $.93(.81)$ & $1.06(.74)$ & $.97(.77)$ \\
\hline PM & $.77(.76)$ & $.92(.78)$ & $.86(.81)$ & $.70(.81)$ & $.82(.79)$ & $.82(.78)$ \\
\hline \multicolumn{7}{|c|}{ Areas } \\
\hline ME & $.48(.80)$ & $.67(.80)$ & $.71(.80)$ & $.68(.76)$ & $.46(.72)$ & $.60(.74)$ \\
\hline $\mathrm{CE}$ & $.40(.78)$ & $.47(.80)$ & $.44(.78)$ & $.55(.88)$ & $.43(.87)$ & $.46(.79)$ \\
\hline MA & $.84(.74)$ & $.68(.77)$ & $1.01(.65)$ & $.77(.78)$ & $.95(.70)$ & $.85(.66)$ \\
\hline
\end{tabular}

Merrill, \& Tannenbaum, 1979; Sherman, Croxton, \& Giovanatto, 1979). Past angle estimation studies (Beery, 1968; Fisher, 1969; MacLean \& Stacey, 1971; MacRae, 1983; MacRae \& Loh, 1981; Wenderoth \& Johnson, 1982) have tended to concentrate on angles lying in the frontal plane and have not applied a power function form to their data; hence, no comparisons can be made with the present data.

\section{Detailed Analyses}

Analyses were performed for each combination of observer, judgment method, metric property, origin, and analysis technique (well over 400 analyses). Instead of reporting all of these (most of which produced no consistent pattern), I report only those patterns for each metric property that were consistent across all combinations. In particular, I report two major results: One pattern is found in distance judgment; the other arises in angle judgment. In both cases, the important variable is stimulus orientation $(\phi)$; the radius (R) and polar angle $(\theta)$ are weak variables that do not influence judgments in any substantial way.

Distances seen in depth (one stake behind the other) tend to be perceptually contracted relative to those in the frontal plane (two stakes side by side). In previous psychophysical work (Baird, 1970), investigators have examined the effects of stimulus orientation on the exponent; here, the pattern arises in the modulus rather than the exponent. The modulus tends to reach a minimum at stake orientations $(\phi)$ of $90^{\circ}$ (in-depth) and maxima at frontal orientations of $0^{\circ}$ and $180^{\circ}$. The modulus increases from this minimum to either maximum in an approximately linear fashion as a function of orientation. Figure 4 displays two examples of the ratio of individual judgments to actual distances (an estimate of the modulus-see the modulus analysis in Appendix A) plotted as a function of the orientation of the corresponding stimulus. The examples show results for two observers, each at a single origin. The pat- tern displayed is typical of the results for other observer, origin, and judgment method combinations, although the exact extent to which in-depth orientations are underestimated is variable. (Figure 4 shows the extremes of compression.) On the average, the same physical distance is seen as slightly more than twice as large in frontal orientations as it is in in-depth orientations for all methods. The binned regression analysis confirms this pattern. No consistent pattern exists in the exponent as a function of orientation. The modulus reaches a minimum at the indepth orientation of $90^{\circ}$ and maxima at frontal orientations of $0^{\circ}$ and $180^{\circ}$.

Angle judgments also show one consistent pattern. As with distance judgments, the pattern arises in the modulus as a function of angle orientation $(\phi)$. The modulus tends to reach maxima at orientations of $-90^{\circ}$ and $90^{\circ}$ (when the angle is facing either directly toward or directly away from the observer) and minima at $-180^{\circ}, 0^{\circ}$, and $180^{\circ}$ (when the angle is facing either to the observer's right or left). Figure 5 presents an example of the modulus analysis as a function of angle orientation. The example shows results for a single observer at a single origin. The pattern displayed is typical of the findings for other observer, origin, and judgment method combinations. The large variability seen in the plots is a consequence of the fact that angle exponents typically deviate significantly from 1.0 (see Appendix A). The binned regression analysis confirms this trend. No consistent pattern exists in the exponent as a function of orientation. The modulus reaches maxima at $-90^{\circ}$ and $90^{\circ}$ and minima at $-180^{\circ}$, $0^{\circ}$, and $180^{\circ}$. There are no exceptions to this trend for either analysis across all combinations of observer, origin, and judgment method.

Both of these results are consistent with the statement that visual space is perceptually contracted in the in-depth dimension relative to the frontal dimension as compared with physical space. Distance judgments directly reflect this hypothesis. Similarly, if angles seen facing directly 


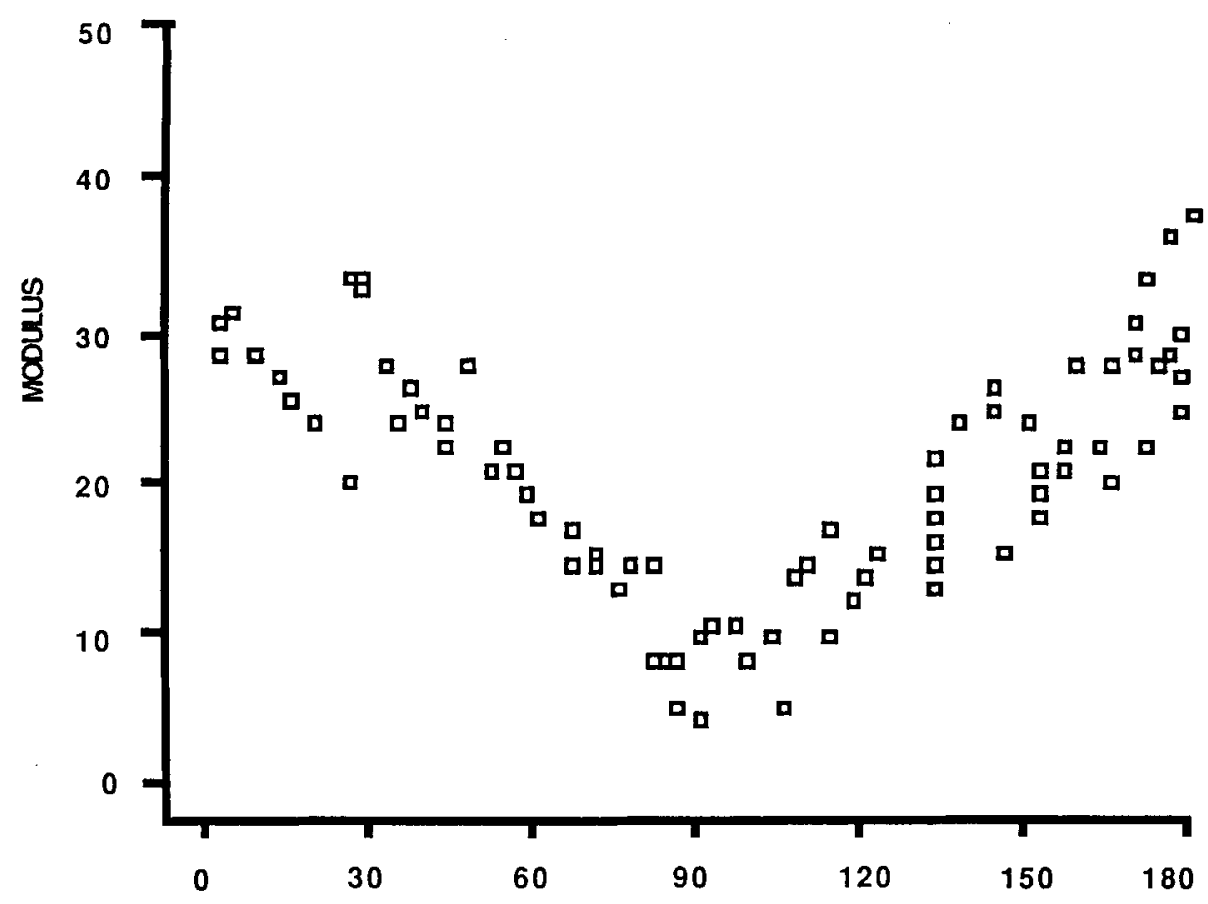

ORIENTATION ANGLE IN DEGREES

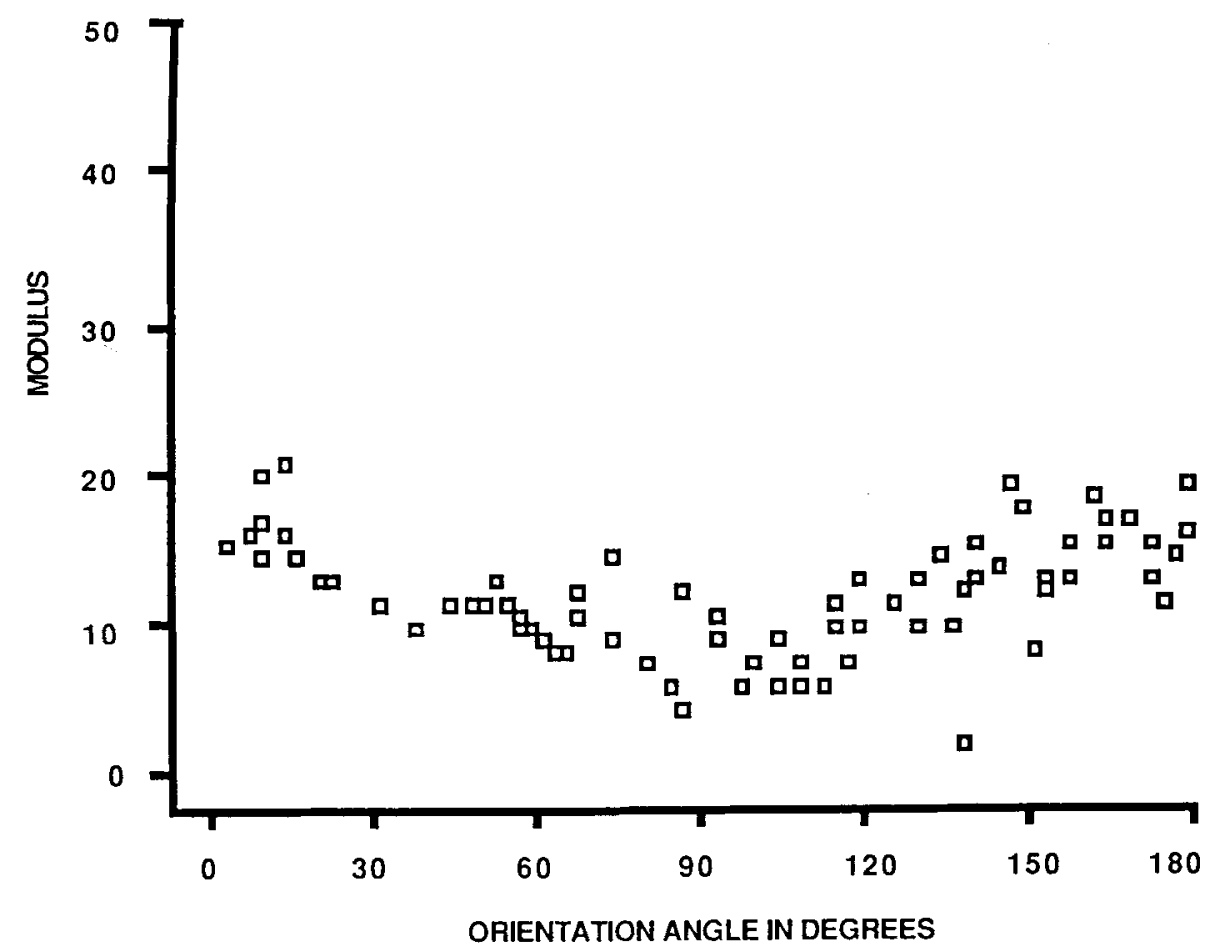

Figure 4. Two examples of an estimate of the modulus plotted as a function of stimulus orientation for distance estimation. Each panel shows the data from one observer, one origin, and one judgment method (magnitude estimation); they were selected to show the extremes of compression. 


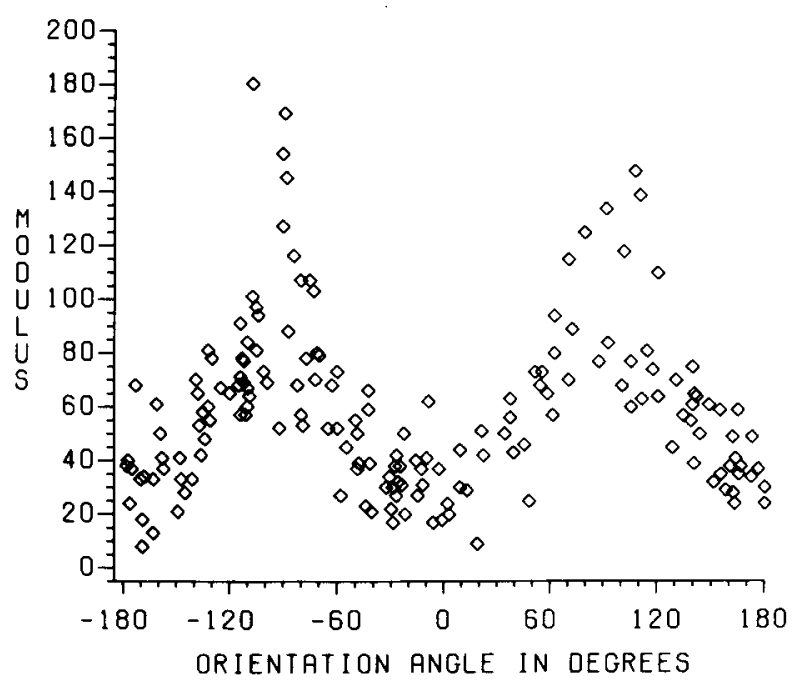

Figure 5. An example of an estimate of the modulus plotted as a function of stimulus orientation for angle estimation. Here $0^{\circ}$ indicates that the angle is facing toward the observer's right; $180^{\circ}$ and $-180^{\circ}$, toward the observer's left; $-90^{\circ}$, toward the observer; and $90^{\circ}$, away from the observer. The data are from one observer and one judgment method (mapping) and are typical of all observers.

toward or away from the observer are "squeezed" in the in-depth dimension, they would perceptually expand in their size. (Points along the sides of the angle remain the same distance apart in the frontal dimension, but are moved closer to the vertex in the in-depth dimension. In the extreme case in which all in-depth distances approach zero, all points would be compressed along a single line, forming a $180^{\circ}$ angle.) Conversely, if angles facing to the observer's right or left are squeezed in the in-depth dimension, they would perceptually contract. This compression of the in-depth dimension (or expansion in the frontal dimension) can be seen in the mapping data. In Figure 6 the actual stake locations are represented by squares and the diamonds show the locations resulting from the mapping data. The $\mathrm{x}$ - and $\mathrm{y}$-dimensions of the plots are to the same scale, and the minimum and maximum x-coordinates for all stake layouts serve as the left and right bounds of the square plot. The minimum and maximum $x$-coordinates across all maps serve as the left and right bounds of the diamond plot. The actual stake location and map location plots are superimposed, and the correspondence between points in the field and on the map are indicated by arrows to allow for direct comparison. The amount of compression in this particular case is extreme. However, quite noticeable compression in the $y-$ dimension is seen in all of the maps.

\section{MATHEMATICAL MODELS}

The preceding data may be used to test the three best known synthetic geometries (Euclidean, spherical, and hyperbolic). Four models based upon these geometries are tested. In the first model, distances in Euclidean physical space are subjected to an affine transformation to produce a somewhat different Euclidean visual space. The second model is a refinement of the first. The third and fourth models test the spherical and hyperbolic geometries using formulations proposed by past theorists. In each case, the model's predictions and the actual responses given by observers were compared by a SIMPLEX curvefitting routine. A variety of initial parameter values were employed to avoid relative minima in the fit. Best-fitting parameter values and measures of goodness of fit are reported.

\section{Affine Contraction Model}

In the first model, the observer is located at the origin of a Cartesian coordinate system, where the $y$-axis corresponds to the direction straight ahead. According to this model, the frontal component of physical space (parallel to the $x$-axis) is unchanged, but the in-depth component (parallel to the $y$-axis) is contracted. This contraction can be expressed as $y^{\prime}=c y$, where $y$ is the physical $y$ coordinate, $y^{\prime}$ is the perceived $y$-coordinate, and $c$ is the contraction constant. After this affine transformation, physical distances and angles are systematically distorted in the resulting visual space. The space is still Euclidean, although it is distorted relative to physical space.

Since distance after an affine transformation is still Euclidean, we may express the distance between two

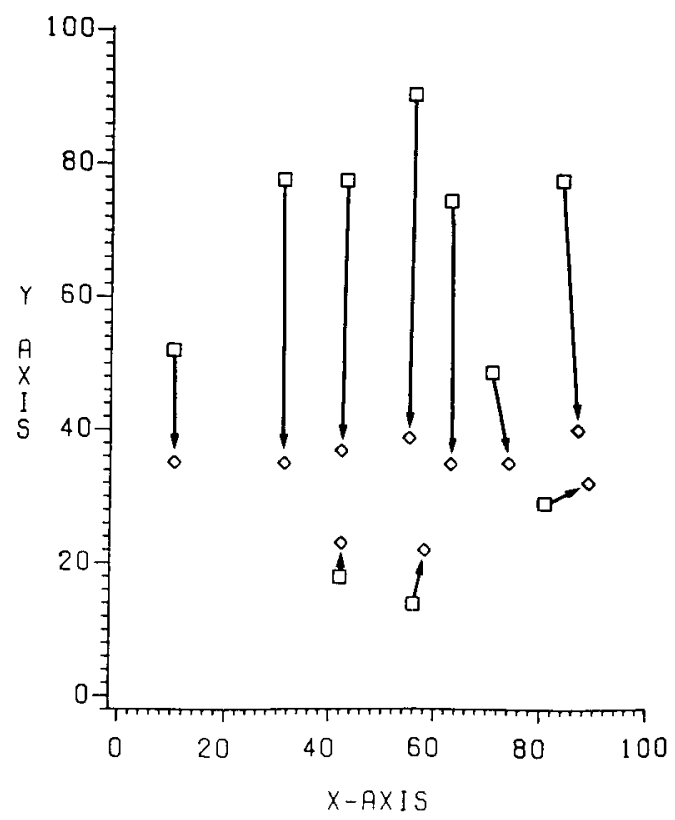

Figure 6. An example of a direct comparison of physical and estimated stake locations for the mapping data. Squares represent the physical locations of the stakes. Diamonds represent the judged location. The arrows display the correspondence between points on the two maps. The data are from one observer and one origin. 
points $\mathrm{P} 1$ and $\mathrm{P} 2$ in the visual world $(\mathrm{J})$ as

$$
\begin{aligned}
J & =\sqrt{\left(x 1^{\prime}-x 2^{\prime}\right)^{2}+\left(y 1^{\prime}-y 2^{\prime}\right)^{2}} \\
& =\sqrt{(x 1-x 2)^{2}+(\operatorname{cy} 1-c y 2)^{2}} \\
& =\sqrt{(x 1-x 2)^{2}+c^{2}(y 1-y 2)^{2}}
\end{aligned}
$$

where $(\mathrm{x} 1, \mathrm{y} 1)$ and $(\mathrm{x} 2, \mathrm{y} 2)$ are the physical coordinates of the points $\mathrm{P} 1$ and $\mathrm{P} 2$ and $\left(\mathrm{x} 1^{\prime}, \mathrm{y} 1^{\prime}\right)$ and $\left(\mathrm{x} 2^{\prime}, \mathrm{y} 2^{\prime}\right)$ are the corresponding visual coordinates.

For modeling purposes, this statement may be generalized to the Minkowski metric:

$$
J=\left\{[x 1-x 2]^{R}+[c(y 1-y 2)]^{R}\right\}^{(1 / R)},
$$

where $R$ is the Minkowski parameter. If $R=2$, the space is Euclidean.

In addition, the data are fit to a power function. In general, the psychophysical literature suggests that the power function accounts for response factors inherent in the judgment method, which gives rise to different exponents and scaling factors. Thus, the general form of the affine contraction model is

$$
J=k\left\{[x 1-x 2]^{R}+[c(y 1-y 2)]^{R}\right\}^{(n / R)},
$$

where $\mathrm{k}$ is a scaling parameter and $\mathrm{n}$ is the psychophysical exponent. The best-fitting parameter values and the overall variance accounted for $\left(r^{2}\right)$, where $r$ is the Pearson product-moment correlation between the model's predictions and the actual data when fit to the distance data for all observers and origins combined, are reported in Table 2.

In general, the fits are quite good. Since only a single set of parameters are fit to the distance data for all observers and origins, individual differences should produce considerable error variance. When the best-fitting curves are derived for each observer and origin separately, the overall variance accounted for is even higher. Regressing the prediction made by the model for each observer and origin against the actual data yields an overall $r^{2}$ of .869 for magnitude estimation, .862 for category estimation, and .929 for mapping.

The Minkowski parameter, $R$, is very close to 2.0 , the Euclidean value. In fact, eliminating the parameter from the fit by setting it equal to 2.0 yields virtually no reduction variance accounted for. The $\mathrm{r}^{2}$ is identical to the one reported in the table to three decimal places.

Table 2

Best-Fitting Parameter Values and the Overall Variance Accounted for, $\mathbf{r}^{2}$, by the Affine Contraction Model When Fit to Distance Judgments Employing Magnitude Estimation (ME), Category Estimation (CE), and Mapping (MA)

\begin{tabular}{cccccc}
\hline & \multicolumn{5}{c}{ Parameter } \\
\cline { 2 - 5 } Method & $\mathrm{k}$ & $\mathrm{n}$ & $\mathrm{R}$ & $\mathrm{c}$ & $\mathrm{r}^{2}$ \\
\hline ME & 26.1 & .97 & 2.22 & .49 & .741 \\
CE & 3.32 & .91 & 1.68 & .53 & .809 \\
MA & 5.93 & .95 & 1.65 & .38 & .793 \\
\hline
\end{tabular}
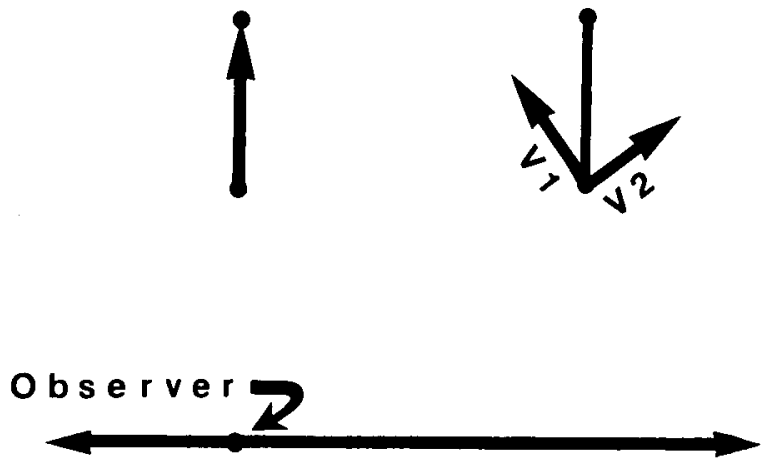

Figure 7. Diagram displaying two pairs of stakes with different orientations with respect to the observer but identical orientation with respect to the $x$-axis. The vectors $v 1$ and $v 2$ are the components into which the distance defined by the pair of stakes on the right are divided in the vector contraction model.

The exponent, $\mathrm{n}$, has a value close to 1.0. The exponent is statistically necessary for category estimation alone. ${ }^{1}$

The contraction parameter, $\mathrm{c}$, is surprisingly small. That is, according to the model, in-depth distances are seen to be about half as large as frontally oriented ones. Eliminating $\mathrm{c}$ by setting it equal to 1.0 , the value expected if depth perception is perfectly veridical, results in large decrements in variance accounted for. Variance accounted for drops from an average of .8 for the full model to .6 for the restricted model, where $c$ is fixed.

Thus, although visual space may be viewed as essentially Euclidean, Gibson $(1950,1959)$ was incorrect in assuming that visual space is exactly equivalent to physical space. Visual space is compressed in the in-depth dimension when compared with physical space (at least within the range of 5-70 $\mathrm{m}$ from the observer).

\section{Vector Contraction Model}

The affine contraction model fits the data quite well. One aspect of the model is unsatisfying, however. It seems unlikely that human beings actually perceive the world in terms of a Cartesian coordinate system. A polar coordinate model seems more appropriate for human experience. An example should clarify this point (see Figure 7). Imagine two stake pairs both of which have the same orientation with respect to the $y$-axis. One of the pairs lies directly in front of the observer. The other lies some distance off to the right. The former stimulus is truly in-depth. One stake lies behind the other in the person's visual field. The latter stimulus will be somewhat frontally oriented. That is, one stake will not lie directly behind the other in the person's visual field. Yet, according to the affine contraction model, both distances should experience the same contraction and be perceived as the same length. Since observers were allowed to move their heads freely in the present experiment, it is unlikely that judgments should be so influenced by an arbitrarily defined Cartesian coordinate system.

The second model modifies the affine contraction model slightly in order to take this problem into account. Im- 
agine a point $\mathrm{P} 1$ lying somewhere in physical space. The distance from this point to some other point P2 may be thought of as a vector originating from P1. The vector is broken down into a frontally oriented component, V1, and an in-depth component, $\mathrm{V} 2$, and the lengths of $\mathrm{V} 1$ and V2 are related to the orientation angle $(\phi)$ of the two stakes with respect to the observer. In particular,

$$
\begin{aligned}
\mathrm{V} 1 & =\mathrm{D} \cos (\phi) \\
\mathrm{V} 2 & =\mathrm{D} \sin (\phi),
\end{aligned}
$$

where $\mathrm{D}$ is the Euclidean distance between the two points. In the vector contraction model, the physical in-depth component V2 is contracted by a constant amount to yield the visual in-depth component $\mathrm{V} 2^{\prime}$. The visual frontal component $\mathrm{Vl}^{\prime}$ is unchanged. Perceived distance $(\mathrm{J})$ is the norm (or length) of this two-component visual vector. This may be written as

$$
\begin{aligned}
\mathrm{J} & =\sqrt{\mathrm{V} 1^{\prime 2}+\mathrm{V} 2^{\prime 2}} \\
& =\sqrt{\mathrm{V} 1^{2}+(\mathrm{cV} 2)^{2}} \\
& =\sqrt{(\mathrm{D} \cos \phi)^{2}+(\mathrm{cDsin} \phi)^{2}} \\
& =\mathrm{D} \sqrt{(\cos \phi)^{2}+(\mathrm{c} \sin \phi)^{2}}
\end{aligned}
$$

This equation can be generalized to the form of a power function

$$
\mathrm{J}=\mathbf{k}\left[\mathrm{D} \sqrt{(\cos \phi)^{2}+(\mathrm{c} \sin \phi)^{2}}\right]^{\mathrm{n}} .
$$

The best-fitting parameter values and the overall variance accounted for by the full model when fit to the distance data with all observers and origins combined are reported in Table 3.

For all observers and origins combined, the vector contraction model accounts for a slightly larger share of the variance than the affine model but is the same in all other respects. The exponent $\mathrm{n}$ tends to be close to one, while the contraction parameter $\mathrm{c}$ is consistently within the range .4 to .5 , indicating that in-depth distances are approximately half as large as frontal distances. If best-fitting curves for each observer and origin are regressed against the data, the overall variance accounted for is .879 for magnitude estimation, .877 for category estimation, and

Table 3

Best-Fitting Parameter Values and the Overall Variance Accounted for, $\mathbf{r}^{2}$, by the Vector Contraction Model When Fit to Distance Judgments Employing Magnitude Estimation (ME), Category Estimation (CE), and Mapping (MA)

\begin{tabular}{ccccc}
\hline & \multicolumn{3}{c}{ Parameter } & $\mathbf{r}^{2}$ \\
\cline { 2 - 5 } Method & $\mathbf{k}$ & $\mathrm{n}$ & $\mathrm{c}$ & .754 \\
ME & 27.6 & .93 & .46 & .834 \\
CE & .33 & .88 & .49 & .802 \\
MA & 6.08 & .93 & .40 & \\
\hline
\end{tabular}

.935 for mapping. In view of the usual amount of psychophysical variability (Baird, 1970), it is unlikely that any other simple model would account for more of the variance. $^{2}$

\section{Spherical Geometry Model}

Thomas Reid (1764/1813) suggested that the eye itself is incapable of depth perception. He stated that "visible" space as such can be represented by a sphere of arbitrary radius encompassing the space. In other words, visual space is exactly equivalent to the proximal stimulus at the retina. This description of visual space is equivalent to a spherical geometry. An example of a spherical geometry is the surface of the earth. "Straight" lines are circles on the surface that contain the earth's center as their own center, such as lines of longitude or the equator. No parallels exist since all lines meet in two locations. This violation of Euclid's parallel postulate is the defining feature of a spherical geometry.

Recently, Angell (1974) and Daniels (1974) have reintroduced Reid's spherical geometry. (More technically, they have proposed a doubly elliptical geometry.) Distance between two points is the length of arc of a great circle passing through the two points, and is proportional to the visual angle between the two points.

A formula for the visual angle in terms of physical measures is simple to derive but involved (see Appendix B). The full spherical model applied to the data is a power function of the form

$$
\mathbf{J}=\mathbf{k}(\xi)^{\mathbf{n}},
$$

where

$$
\xi=\operatorname{Arccos}\left[\frac{h^{2}+R^{2}+R D \sin \phi}{\sqrt{R^{2}+h^{2}} \sqrt{R^{2}+D^{2}+h^{2}+2 R D \sin \phi}}\right],
$$

where $J$ is the judgment, $k$ is a scaling factor, $n$ is the exponent, $\xi$ is the visual angle between the two points, $R$ is the radius to one of the points, $D$ is the physical distance between the two points in Euclidean space, $\phi$ is the orientation angle, and $h$ is the height of the observer above the plane in which the stakes lie.

The overall variance accounted for and the best-fitting parameter values of this model when fit to the distance data with all observers and origins combined are reported in Table 4. In all cases, the height of the observer is absurdly high. Setting the parameter to a reasonable height of $2.0 \mathrm{~m}$ yields a large decrement in variance accounted for. Because of this and the relatively poor fits, the spherical model is rejected. ${ }^{3}$

\section{Hyperbolic Geometry Model}

Luneburg $(1947,1950)$ proposed that visual space is best described as a hyperbolic geometry. A hyperbolic geometry can be thought of as the inverse of an elliptical geometry. Here, an infinite number of parallels can be drawn to a line through a point not on that line. 
Table 4

Best-Fitting Parameter Values and the Overall Variance Accounted for, $\mathbf{r}^{2}$, by the Spherical Geometry Model When Fit to Distance Judgments Employing Magnitude Estimation (ME), Category Estimation (CE), and Mapping (MA)

\begin{tabular}{crccc}
\hline & \multicolumn{3}{c}{ Parameter } & \\
\cline { 2 - 4 } Method & \multicolumn{1}{c}{$\mathrm{k}$} & $\mathrm{n}$ & $\mathrm{h}$ & $\mathrm{r}^{2}$ \\
\hline ME & 2203.5 & 1.0 & $108.5 \mathrm{~m}$ & .603 \\
CE & 17.4 & .91 & $92.5 \mathrm{~m}$ & .711 \\
MA & 276.2 & .92 & $53.0 \mathrm{~m}$ & .659 \\
\hline
\end{tabular}

One of the most recent formulations (Indow, 1974) for this function for two points, $P 1$ and $P 2$, is

$\mathrm{J}=\mathrm{k}\{(\mathrm{T}) \operatorname{arcsinh}[\sqrt{[1+\mathrm{K}(\mathrm{q} 1) / 4][1+\mathrm{K}(\mathrm{q} 2) / 4]}(1 / \mathrm{T}) \mathrm{D}]\}^{\mathrm{n}}$,

where $\mathrm{q}_{\mathrm{i}}=2 \exp [-\mathrm{aR}(\mathrm{i})]$ and $\mathrm{T}=2 / \sqrt{-\mathrm{K}}$, where $\mathrm{J}$ is the observer's judgment, $k$ is a scaling parameter, arcsinh is the inverse hyperbolic sine, $\mathrm{q} 1$ and $\mathrm{q} 2$ are the visual radii from the origin to $P 1$ and $P 2$, respectively, $R(i)$ is related to the distance from the observer to the points (more precisely, it is the convergence angle of the two eyes), $\mathrm{D}$ is physical distance between the two points scaled relative to the $q_{i} s$, $a$ is a personal constant for each observer, and $\mathrm{K}$ is the Riemannian curvature of the space. In this model, curvature is assumed to be constant. In general, spaces of constant curvature are of three types. If $\mathrm{K}>0$, the space is elliptical (as in the spherical geometry model); if $\mathrm{K}=0$, the space is Euclidean; and if $\mathrm{K}<0$, the space is hyperbolic. Equation 2 applies only to the hyperbolic case. If visual space were Euclidean, the formula would require division by zero. If visual space were elliptical, the formula would require calculations with imaginary numbers. Neither situation is desirable from a curve-fitting standpoint.

Fitting the hyperbolic model to the distance data proved to be problematic. In spite of repeated attempts with different starting values for the parameters (to avoid relative minima problems), a complete run of the curve-fitting routine was seldom possible. The program tended to "crash" as the parameter estimate for curvature approached zero or as $\sqrt{[1+K(q 1) / 4][1+K(q 2) / 4]}$ became imaginary. The few definite solutions that occurred were relative minima in the curve-fitting procedure that accounted for almost none of the variance in the data. ${ }^{4}$

Hence, the present results are not consistent with work by Luneburg (1947), Blank (1959, 1978), and Indow $(1974,1979)$. Either visual space is not hyperbolic or Luneburg's mapping functions between visual and physical space are incorrect. There are at least three possible reasons for this inconsistency: (1) In an attempt to employ ecological viewing conditions, both free head and free eye movement were allowed in the present study. Luneburg allowed only free eye movement. (2) Luneburg's and Blank's theories were based upon binocular cues to distance. Such cues are limited in scope-useful only up to $2 \mathrm{~m}$ away from the observer (Baird, 1970). Thus, their hyperbolic models are also limited in scope. Indow, Inoue, and Matsushima (1963) found that certain predictions of the hyperbolic model were somewhat "disappointing" when applied to a spacious field. (3) Probably the most important difference between this work and Luneburg's is the conditions under which stimuli were observed. The present study took place under full-cue conditions. That is, the experiment took place under natural conditions with good lighting and plenty of textural cues to depth. In contrast, Luneburg's stimuli were luminous points in the dark, eliminating most of the major cues to depth. Battro, Netto, and Rozestraten (1976) have disconfirmed a number of the hyperbolic model's predictions under full-cue settings. In particular, the curvature constant $(\mathrm{K})$ varied widely between -1 and +1 , a result that is inconsistent with the hyperbolic model.

\section{Angle Data}

The affine contraction model. The affine contraction and the vector contraction models may be fit to the angle data as well as to the distance data. For a Euclidean geometry,

$$
\cos \left(A^{\prime}\right)=\left(v^{\prime} \cdot w^{\prime}\right) /\left(\left\|v^{\prime}\right\|\left\|w^{\prime}\right\|\right) .
$$

For the affine contraction model, the power function formulation is

$$
\mathrm{A}^{\prime}=\mathrm{k}\left\{\arccos \left[\left(\mathrm{v}^{\prime} \cdot \mathbf{w}^{\prime}\right) /\left(\left\|\mathrm{v}^{\prime}\right\|\left\|\mathbf{w}^{\prime}\right\|\right)\right]\right\}^{\mathrm{n}}
$$

and

$$
\begin{aligned}
\left(v^{\prime} \cdot w^{\prime}\right) & =v 1 w 1+c^{2} v 2 w 2 \\
\left\|v^{\prime}\right\| & =\sqrt{v 1^{2}+(c v 2)^{2}} \\
\left\|w^{\prime}\right\| & =\sqrt{w 1^{2}+(c w 2)^{2}},
\end{aligned}
$$

where $\mathrm{k}$ is a scaling factor, $\mathrm{n}$ is the exponent, $\mathrm{v} 1$ and $\mathrm{v} 2$ are the $\mathrm{x}$ - and $\mathrm{y}$-compenents of the vector $\mathrm{v}$ in physical space, $w 1$ and $w 2$ are the $x$ - and $y$-components of the vector $w$ in physical space, and $c$ is the contraction factor of the affine contraction model.

Table 5 reports the best-fitting parameter values and variance accounted for by the model with all observers and origins combined. The exponent $\mathbf{n}$ is much less than 1.0 in all cases. The exponents obtained for each judgment method are close to the overall exponents reported in the results section. The contraction parameter, $c$, is much less than the veridical value of 1.0. Eliminating $\mathrm{c}$ by setting it equal to 1.0 results in a decline in variance accounted for from the average of .72 for the full model to .62 .

The magnitude of the compression is similar to that found for the affine model when it is applied to the distance data ( $c$ ranges between .4 and .6). Replacing the contraction parameter by the values found in Table 2 for distance estimation produced little decrement in variance accounted for. The $r^{2}$ for category estimation and map- 
Table 5

Best-Fitting Parameter Values and the Overall Variance Accounted for, $r^{2}$, by the Affine Contraction Model When Fit to Angle Judgments Employing Magnitude Estimation (ME), Category Estimation (CE), Mapping (MA), and Perceptual Matching (PM)

\begin{tabular}{crccc}
\hline & \multicolumn{3}{c}{ Parameter } & \\
\cline { 2 - 5 } Method & \multicolumn{1}{c}{$\mathrm{k}$} & $\mathrm{n}$ & $\mathrm{c}$ & $\mathrm{r}^{2}$ \\
\hline ME & 71.90 & .79 & .63 & .700 \\
CE & 3.31 & .46 & .52 & .703 \\
MA & 55.30 & .86 & .39 & .725 \\
PM & 5.60 & .72 & .39 & .747 \\
\hline
\end{tabular}

ping were identical to the full model's out to three decimal places. For magnitude estimation, a slight decline in variance accounted for from .70 for the full model to .69 is evident. Notice that a fourth judgment method, perceptual matching, produced a similar parameter value.

Thus, the affine contraction model produces good fits to the angle data as well as to the distance data. Parameter values are similar and do not support the usual idea that visual space directly reflects physical space $(c=1.0)$.

Vector contraction model. The same formula (which applies to all Riemannian geometries) may be applied to obtain the judged angle for the vector contraction model. That is,

$$
A^{\prime}=k\left\{\arccos \left[\left(v^{\prime} \cdot w^{\prime}\right) /\left\|v^{\prime}\right\|\left\|w^{\prime}\right\|\right]\right\}^{n}
$$

and

$$
\begin{aligned}
v^{\prime} \cdot w^{\prime}= & {[D(v) \cos \phi)][D(w) \cos \phi(v)] } \\
& +[\mathrm{cD}(v) \sin \phi(v)][c D(w) \sin \phi(w)] \\
\left\|v^{\prime}\right\|= & \sqrt{[D(v) \cos \phi(v)]^{2}+[c D(v) \sin \phi(v)]^{2}} \\
\left\|w^{\prime}\right\|= & \sqrt{[D(w) \cos \phi(w)]^{2}+[c D(w) \sin \phi(w)]^{2}},
\end{aligned}
$$

where $v^{\prime}$ and $w^{\prime}$ are the two visual vectors corresponding to the two sides of the angle, $D(v)$ and $D(w)$ are the lengths of the two sides in physical space, $\phi(v)$ and $\phi(w)$ are the orientation angles of the two sides, $\mathrm{k}$ is a scaling factor, $\mathrm{n}$ is the exponent, and $\mathrm{A}^{\prime}$ is the judged angle.

The best-fitting parameter estimates, along with their associated measures of goodness of fit, are presented in Table 6. The vector contraction model fits the data either as well as or slightly better than the affine contraction model. The contraction parameter falls in the same range found for the distance data. When the contraction parameter for angle and distance estimation are compared in the context of the alternative that there is no contraction in the space $(c=1.0)$, then any differences between the parameters produced by the various methods and the type of object judged (distance or angle) seem small indeed. Overall, the affine contraction and vector contraction models are strongly supported by the angle data.

\section{DISCUSSION}

The present data indicate that visual space is severely compressed in the in-depth dimension relative to physical space. This conclusion is consistent across observers, origins, judgment methods, and analysis (modeling) techniques. The mathematical form of this compression appears to be some variant of an affine transformed Euclidean space. The extent of the compression is large. The same physical distance will be seen as approximately twice as large when viewed in frontal orientations as when viewed in depth.

One interesting aspect of these orientation effects is that they are carried by the modulus. Modulus effects are rare in the psychophysical scaling of large-scale visual space but do arise periodically in other areas of psychophysical investigation (Babkoff, 1976; Crawford, McDonald, \& Hilgard, 1979; J. C. Stevens \& Marks, 1980; M. Teghtsoonian, 1972, 1980). The meanings of modulus and exponent effects are slightly different, as can be seen by examining their role in the power function. Exponents different from 1.0 indicate perceptual and judgment factors that differentially affect judgments of large and small stimuli. Modulus changes indicate factors that affect all judgments proportionally the same for all sizes of stimuli. Hence a modulus that is smaller than under the standard condition means that stimuli are in general seen to be smaller under the new condition than under the standard condition; that is, the modulus reveals a consistent illusion for stimuli of all sizes. Borg and Marks (1983) report a more detailed analysis of the meaning of modulus effects.

Modeling visual space as an affine-transformed Euclidean space appears to contradict work by such previous investigators as Gibson, Indow, Luneburg, and Reid. This contradiction is probably only one of appearance, however. All of the theories adequately described data collected under an appropriate set of environmental conditions. The conditions examined were different for each theorist, however. Gibson was concerned with well-lit settings in which the observer was allowed full freedom of motion. The present data were collected under full-cue conditions with free head movement but limited body

Table 6

Best-Fitting Parameter Values and the Overall Variance Accounted for, $r^{2}$, by the Vector Contraction Model When Fit to Angle Judgments Employing Magnitude Estimation (ME), Category Estimation (CE), Mapping (MA), and Perceptual Matching (PM)

\begin{tabular}{crccc} 
& \multicolumn{3}{c}{ Parameter } & \\
\cline { 2 - 5 } Method & \multicolumn{1}{c}{$\mathrm{k}$} & $\mathrm{n}$ & $\mathrm{c}$ & $\mathbf{r}^{2}$ \\
\hline ME & 71.40 & .81 & .65 & .699 \\
CE & 3.30 & .45 & .47 & .719 \\
MA & 56.00 & .85 & .38 & .727 \\
PM & 5.90 & .67 & .30 & .794 \\
\hline
\end{tabular}


movement. Luneburg's data were collected in the dark using methods of adjustment and no head movement. Reid based his work on observations made without free eye movements. In sum, this multiplicity of well-supported theories indicates that no single geometry can adequately describe visual space under all conditions. Instead, the geometry of visual space itself appears to be a function of stimulus conditions. Other theorists have reached similar conclusions (Dodwell, 1982; Suppes, 1977; Watson, 1978).

In general, the visual world approaches the Euclidean ideal of veridical perception as the quantity and quality of perceptual information increases. Thus far, most of the reported geometries have been Riemannian with constant curvature. One might speculate that the primary effect of the environmental setting is to influence the curvature constant varying from $\mathrm{K}=1$ under totally reduced settings with "projective" estimation instructions to $\mathrm{K}=0$ under information-rich settings with "objective" estimation instructions.

This speculation is essentially equivalent to the general finding in the direct estimation and constancy literatures that judgments of size range between matching the visual angle of the stimulus under reduced-cue conditions using projective estimation instructions and matching the physical size of the stimulus under full-cue conditions using objective estimation instructions (Baird, 1970).

How do the present data fit into the above scheme? One possibility is that the curvature reflected in the present data lies beween 0 and 1 and that frontally oriented distances are seen as longer by virtue of subtending a larger visual angle than in-depth oriented distances at the same radial distance from the observer. The second possibility is that in the absence of known size cues to anchor judgments, in-depth and frontal dimensions may be scaled differently. The former possibility is unlikely. Simulations demonstrate that the ratio of frontal to in-depth visual angles increase dramatically as a function of distance from the observer $(R)$. No distance effects were discerned in the present data. Five attempts to model visual space taking projective size into account failed. The second possibility is supported by the linear decrease in judged size as a function of orientation found in the data and by the good fits obtained by the affine and vector contraction models.

\section{REFERENCES}

ANGELl, R. B. (1974). The geometry of visibles. Nous, 8, 87-117. BABKOFF, H. (1976). Magnitude estimation of short electrocutaneous pulses. Psychological Research, 39, 39-49

BAIRD, J. C. (1970). Psychophysical analysis of visual space. Oxford Pergamon Press.

Baird, J. C., Merrill, A. A., \& Tannenbaum, J. (1979). Cognitive representation of spatial relations: II. A familiar environment. Journal of Experimental Psychology: General. 108, 92-98.

BAIRD. J. C. . \& NomA. E. (1978).. Fundamentals of scaling and psychophysics. New York: Wiley.

Baird, J. C., WaGNER, M., \& Noma, E. (1982). Impossible cognitive spaces. Geographical Analysis, 14, 204-216.
Battro, A. M., Netto, S. P., \& Rozestraten, R. J. A. (1976). Riemannian geometries of variable curvature in visual space: Visual alleys, horopters, and triangles in big open fields. Perception, 5, 9-23.

BeERY, K. E. (1968). Estimation of angles. Perceptual \& Motor Skills, 26, $11-14$.

BLANK, A. A. (1953). The Luneburg theory of binocular visual space. Journal of the Optical Society of America, 43, 717-727.

BLANK, A. A. (1959). The Luneburg theory of binocular space perception. In S. Koch (Ed.), Psychology: A study of a science (Vol. 1, pp. 395-426). New York: McGraw-Hill.

BLANK A. A. (1978). Metric geometry in human binocular perception: Theory and fact. In E. L. J. Leeuwenberg \& H. F. J. M. Buffart (Eds.), Formal theories of visual perception (pp. 83-102). New York: Wiley.

Borg, G. A., \& MARKs, L. E. (1983). Twelve meanings of the measure constant in psychophysical power functions. Bulletin of the Psychonomic Society, 21, 73-75.

BRUNSwIK, E. (1944). Distal focusing of perception. Psychological Monographs, 56, 1-48.

Carlson, V. R. (1977). Instructions and perceptual constancy judgments. In W. Epstein (Ed.), Stability and constancy in visual perception (pp. 217-254). New York: Wiley.

Crawford, H. J., MacDonald, H., \& Hilgard, E. R. (1979). Hypnotic deafness: A psychophysical study of responses to tone intensity as modified by hypnosis. American Journal of Psychology, 92, 193-214.

Daniels, N. (1974). Thomas Reid's inquiry. New York: Burt Franklin. DODWELL, P. C. (1982). Geometrical approaches to visual processing. In D. J. Ingel, A. M. Goodale, \& R. J. W. Mansfield (Eds.), Analysis of visual behavior (pp. 217-254). Cambridge, MA: MIT Press.

Fisher, G. H. (1969). An experimental study of angular substension. Quarterly Journal of Experimental Psychology, 21, 356-366.

GiBson, J. J. (1950). The perception of the visual world. Boston: Houghton Mifflin.

GibSON, J. J. (1959). Perception as a function of stimulation. In S. Koch (Ed.), Psychology: A study of a science (Vol. 1, pp. 459-501). New York: McGraw-Hill

Hoffman, W. C. (1966). The Lie algebra of visual perception. Jour nal of Mathematical Psychology, 3, 65-98.

Hoffman, W. C. (1980). Subjective geometry and geometric psychology. Mathematical Modeling. 1, 349-367.

INDow, T. (1967). Two interpretations of binocular visual space: Hyperbolic and Euclidean. Annals of the Japanese Association for Physiological Science. 3, 51-64.

INDOw, T. (1974). On geometry of frameless binocular perceptual space Psychologia, 17, 50-63.

INDow. T. (1979). Alleys in visual space. Journal of Mathematical Psychology, 19, 221-258.

Indow. T., Inoue, E., \& Matsushima, K. (1963). An experimental study of the Luneburg theory of binocular space perception (3), the experiments in a spacious field. Japanese Psychological Research, 5. $1-27$

Leibowitz, H. W., \& Harvey, L. O., Jr. (1969). Effect of instructions, environment, and type of test on matched size. Journal of $E x$ perimental Psychology, 81, 36-43.

LUNEBURG, R. K. (1947). Mathematical analysis of binocular vision. Princeton, NJ: Princeton University Press.

LUNEBurg, R. K. (1948). Metric methods in binocular visual perception: Studies and essays. Courant Anniversary Volume, 215-240.

LUNEBURG, R. K. (1950). The metric of binocular visual space. Journal of the Optical Society of America, 40, 637-642.

MacLean, I. E., \& Stacey, B. G. (1971). Judgment of angle size: An experimental appraisal. Perception \& Psychophysics, 9, 499-504

MACRAE, A. W. (1983). Constant errors in the simultaneous matching of angles are not an artifact of the starting position for adjustment. Perception \& Psychophysics, 33, 96-98.

MaCRAE, A. W. \& LoH, H. D. (1981). Constant errors occur in matched reproduction of angles even when likely biases are eliminated. Perception \& Psychophisics, 30, 341-346.

REID. T. (1813). Inquiry into the human mind. In D. Stewart (Ed.), 
Reid's works (Vol. 1). Charlestown, WV: Samuel Etheridge. (Original work published 1764)

Sherman, R. C., Croxton, J., \& Giovanatto, J. (1979). Investigating cognitive representations of spatial relationships. Environment \& Behavior, 11, 209-226.

Stevens, J. C., \& HaLl, J. W. (1966). Brightness and loudness as functions of stimulus duration. Perception \& Psychophysics, 1, 319-327.

Stevens, J. C., \& Marks, L. E. (1980). Cross-modality matching functions generated by magnitude estimation. Perception \& Psychophysics, 27, 379-389.

Stevens, J. C., \& Rubin, L. L. (1970). Psychophysical scales of apparent heaviness and the size-weight illusion. Perception \& Psychophysics, 8, 225-230.

Stevens, S. S. (1975). Psychophysics. New York: Wiley.

SUPPES, P. (1977). Is visual space Euclidean? Synthese, 35, 397-421.

Teghtsoonian, M. (1972). Apparent length as a function of tilt does not depend on orientation of the standard. Journal of Experimental Psychology, 94, 191-197.

Teghtsoonian, M. (1980). Children's scales of length and loudness: A developmental application of cross-modality matching. Journal of Experimental Child Psychology, 30, 290-307.

Teghtsoonian, M., \& Teghtsoonian, R. (1969). Scaling apparent distance in a natural indoor setting. Psychonomic Science, 16, 281-283.

Teghtsoonian, R., \& Teghtsoonian, M. (1970). Scaling apparent distance in a natural outdoor setting. Psychonomic Science, 21, 215-216.

Teghtsoonian, R., \& Teghtsoonian, M. (1978). Range and regression effects in magnitude scaling. Perception \& Psychophysics, 24, 305-314.

WAGNER, M. (1982). The metric of visual space. Unpublished doctoral dissertation, Dartmouth College.

Watson, A. (1978). A Riemannian geometric explanation of the visual illusions and figural aftereffects. In F. L. J. Leeuwenberg \& H. F. J. M. Buffart (Eds.), Formal theories of visual perception. New York: Wiley.

Wenderoth, P., \& Johnson, M. (1982). Constant errors do not occur in the matched reproduction of angles. Perception \& Psychophysics, 32, 388-392.

\section{NOTES}

1. All statements made in the text about variance accounted for by a parameter are supported by statistical tests. Statistically significant reductions in variance accounted for by eliminating a parameter indicate that the parameter is statistically "necessary." For the sake of brevity and clarity, these tests are omitted in the text. The interested reader may refer to Wagner (1982) for further details.

2. Two other types of depth contraction models failed to describe the experimental data. These models are described in Wagner (1982).

3. Three other visual angle models are reported in Wagner (1982). These models also predict that the observer should be too tall.

4. A second hyperbolic model is reported in Wagner (1982). In this model, physical distances between points are directly substituted into the hyperbolic equation instead of angular measures. In all cases, curvature approached or equaled zero, indicating a Euclidean space.

\section{APPENDIX A}

The binned regression technique attempts to determine how $k$ and $n$ vary as a function of $R, \theta$, and $\phi$. A coordinate dimension such as $R$ is divided into regions (bins). Every stimulus pair satisfying the defining feature of a bin (e.g., being between 10 and $15 \mathrm{~m}$ away or being between 15 and $20 \mathrm{~m}$ away) has its $\log$ distance and $\log$ estimate computed. Now, all such pairs for the bin are regressed simultaneously, yielding a slope and an intercept. The slope represents the exponent for that bin, and the intercept represents the log modulus.
This technique will not produce a continuous scale. One exponent and one modulus is calculated for each bin. Of course, the number of points generated will depend upon the number of bins used and upon the size of each bin. A tradeoff exists between fine-grained analysis and stability. Larger bins (a coarser analysis) will contain more points and yield less variable parameter estimates.

The second approach (the modulus analysis) plots the ratio of the judgment to the actual distance as a function of one of the coordinate dimensions such as $\mathrm{R}$. Mathematically, this ratio provides a reasonable estimate for $\mathrm{k}$. For given values of $\mathrm{R}, \theta$, and $\phi$,

$$
\mathbf{J}=\mathbf{k}^{\prime} \mathbf{D}^{\mathbf{n}^{\prime}}
$$

where $\mathrm{k}^{\prime}$ and $\mathrm{n}^{\prime}$ are constants for each given value for $\mathrm{R}, \theta$, and $\phi$.

Dividing both sides by $\mathrm{D}$ yields

$$
\begin{aligned}
J / D & =\left(k^{\prime} D^{n^{\prime}}\right) / D \\
& =k^{\prime} D^{\left(n^{\prime}-1\right)}
\end{aligned}
$$

If $\mathrm{n}^{\prime}$ is close to 1 (which it tends to be for distance), the ratio of judgment to distance is approximately equal to $\mathrm{k}$. Any variability that does exist in $D^{\left(n^{\prime}-1\right)}$ will act as variability in the estimate of $k$.

This technique has several advantages. First, since every judgment can generate a ratio of this sort, a large number of estimates for k can be obtained. Second, since this technique does not require data binning of any sort, a reasonably continuous scale results.

There are several problems with this technique, however. First, the basic rationale is to minimize the influence of $n^{\prime}$ and $D^{\prime}$, but it does not eliminate them. Significant variance in $n^{\prime}$ departing from 1.0 will introduce our second variable of interest, $\mathbf{n}^{\prime}$, into the equation. Thus, this method must be used with caution and in conjunction with the binned regression technique, which can disambiguate whether effects are due to the modulus or the exponent.

\section{APPENDIX B}

We are trying to find the visual angle, $\xi$, defined by two points lying on a plane, P1 and P2, in terms of the known quantities, $h$, the height of the observer, $R$, the distance to the nearest point, $D$, the Euclidean distance between $P 1$ and $P 2$, and $\phi$, the orientation angle (see diagram).

By the law of cosines,

$$
g^{2}=R^{2}+D^{2}-2 R D \cos (90+\phi) .
$$

where $g$ is the distance along the ground from point $G$ (at the observer's feet) to point P2.

By the Pythagorean formula,

$$
Z 2^{2}=g^{2}+h^{2}
$$

where $\mathrm{Z} 2$ is the distance from the observer's eyes $(\mathrm{O})$ to $\mathrm{P} 2$. By the Pythagorean formula.

$$
\mathrm{ZI}^{2}=\mathrm{R}^{2}+\mathrm{h}^{2}
$$

where $\mathrm{Zl}$ is the distance from the observer's eyes $(\mathrm{O})$ to $\mathrm{Pl}$. 


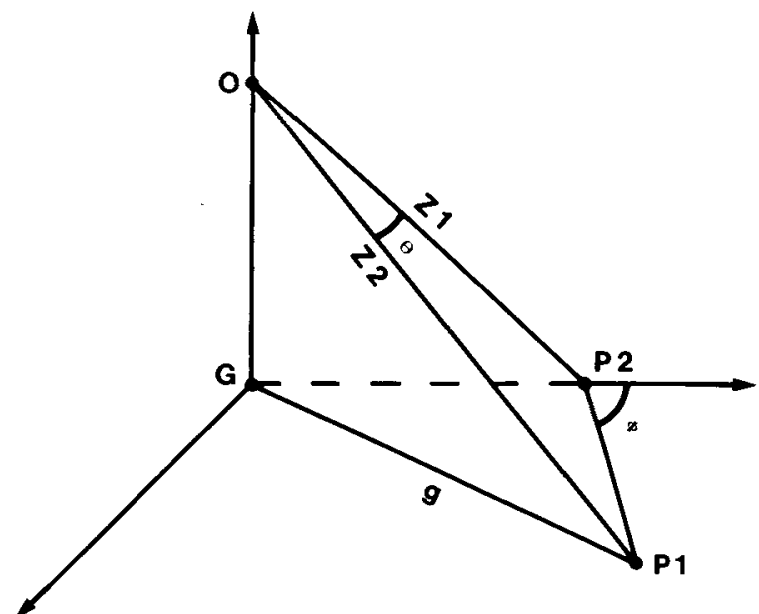

By the law of cosines

$$
\begin{aligned}
\mathrm{D}^{2} & =\mathrm{Zl}^{2}+\mathrm{Z} 2^{2}-2 \mathrm{Z} 1 \mathrm{Z} 2 \cos (\xi) \\
& =\left(\mathrm{R}^{2}+\mathrm{h}^{2}\right)+\left(\mathrm{g}^{2}+\mathrm{h}^{2}\right)-2 \sqrt{\mathrm{R}^{2}+\mathrm{h}^{2}} \sqrt{\mathrm{g}^{2}+\mathrm{h}^{2}} \cos (\xi)
\end{aligned}
$$

$$
\begin{aligned}
= & \left(\mathbf{R}^{2}+\mathrm{h}^{2}\right)+\left[\mathrm{R}^{2}+\mathrm{D}^{2}-2 \mathrm{RD} \cos (90+\phi)+\mathrm{h}^{2}\right] \\
& -2 \sqrt{\mathrm{R}^{2}+\mathrm{h}^{2}} \sqrt{\mathrm{g}^{2}+\mathrm{h}^{2}} \cos (\xi) .
\end{aligned}
$$

This may be reexpressed as

$$
\begin{aligned}
& 2 \sqrt{\mathrm{R}^{2}+\mathrm{h}^{2}} \sqrt{\mathrm{g}^{2}+\mathrm{h}^{2}} \cos \xi=2 \mathrm{R}^{2}+2 \mathrm{~h}^{2}-2 \mathrm{RD} \cos (90+\phi) \\
& \cos \xi=\frac{\mathrm{R}^{2}+\mathrm{h}^{2}-\mathrm{RD} \cos (90+\phi)}{\sqrt{\mathrm{R}^{2}+\mathrm{h}^{2}} \sqrt{\mathrm{R}^{2}+\mathrm{D}^{2}-2 \mathrm{RD} \cos (90+\phi)+\mathrm{h}^{2}}} . \\
& \text { Since }-\cos (90+\phi)=\sin \phi, \text { we have } \\
& \xi=\operatorname{Arccos}\left[\frac{\mathrm{R}^{2}+\mathrm{h}^{2}+\mathrm{RD} \sin \phi}{\sqrt{\mathrm{R}^{2}+\mathrm{h}^{2}} \sqrt{\mathrm{R}^{2}+\mathrm{D}^{2}+\mathrm{h}^{2}+2 \mathrm{RD} \sin \phi}}\right],
\end{aligned}
$$

which was what we set out to prove.

(Manuscript received December 10, 1984;

revision accepted for publication October 15, 1985.) 